Research Paper

\title{
Red Cell Distribution Width (RDW) and Complete Blood Cell Count-Derived Measures in Non-Arteritic Anterior Ischemic Optic Neuropathy
}

\author{
Antonio Pinna ${ }^{1,2}{ }^{\varpi}$, Paola Carlino ${ }^{1}$, Rita Serra ${ }^{3}$, Francesco Boscia ${ }^{4}$, Stefano Dore ${ }^{1}$, Ciriaco Carru ${ }^{2,3}$, Angelo \\ Zinellu ${ }^{3}$ \\ 1. Department of Medical, Surgical, and Experimental Sciences, University of Sassari, Sassari, Italy. \\ 2. Azienda Ospedaliero-Universitaria di Sassari, Sassari, Italy. \\ 3. Department of Biomedical Sciences, University of Sassari, Sassari, Italy. \\ 4. Section of Ophthalmology, Department of Basic Medical Science, Neuroscience and Sense Organs, University of Bari, Bari, Italy. \\ $\triangle$ Corresponding author: Prof. Antonio Pinna, Department of Surgical, Microsurgical, and Medical Sciences, Section of Ophthalmology, University of Sassari, \\ Viale San Pietro 43 A, 07100 Sassari, Italy. Telephone: +39079229141; E-mail: apinna@uniss.it. \\ (C) The author(s). This is an open access article distributed under the terms of the Creative Commons Attribution License (https://creativecommons.org/licenses/by/4.0/). \\ See http://ivyspring.com/terms for full terms and conditions.
}

Received: 2020.09.24; Accepted: 2020.12.18; Published: 2021.03.27

\begin{abstract}
Purpose: To assess the role of complete blood cell count (CBC) dimensional indices and CBC-derived measures in non-arteritic anterior ischemic optic neuropathy (NA-AION).

Methods: In this retrospective case-control survey, 37 newly diagnosed NA-AION patients and 37 sexand age-matched cataract controls were enrolled in 2017-2018. On the same day of NA-AION diagnosis, a blood sample was collected and $\mathrm{CBC}$ was determined using an automatic blood counter. CBC dimensional indices, such as mean platelet volume (MPV) and red cell distribution width (RDW), and CBC-combined indices, including neutrophil/lymphocyte ratio (NLR), derived NLR [dNLR = neutrophils/(white blood cells - neutrophils)], and platelet/lymphocyte ratio (PLR), were evaluated. Erythrocyte sedimentation rate (ESR) was also measured.

Results: Mean platelet count, median MPV, RDW, NLR, and dNLR were $221 \pm 48 \times 109 / \mathrm{L}, 8.2 \mathrm{fL}$ (IQR=7.6-8.9), 13\% (IQR=12-14.5), $2.50 \quad(\mathrm{IQR}=1.77-3.06)$, and 1.73 (IQR=1.31-2.07) in NA-AION patients and $248 \pm 56 \times 10 \% / \mathrm{L}, 7.60 \mathrm{fL}(\mathrm{IQR}=7.05-8.25), 12 \%(\mathrm{IQR}=11.6-13), 1.95(\mathrm{IQR}=1.43-2.49)$ and 1.36 $(I Q R=1.07-1.69)$ in controls. NA-AION patients showed significantly lower platelet count $(p=0.03)$ and significantly higher median values of MPV $(p=0.01), \operatorname{RDW}(p=0.015), \operatorname{NLR}(p=0.03)$, and dNLR $(p=0.01)$. Multivariate logistic regression models disclosed a significant correlation only between higher levels of RDW and NA-AION $(\mathrm{p} \leq 0.05)$. The attributable risk of the association between NA-AION and RDW was $33 \%$.

Conclusions: Results suggest that RDW may be somehow involved in the pathogenesis of NA-AION. However, high-quality cohort studies are warranted to confirm whether, or not, an altered RDW may be considered a potential biomarker of this vascular disorder affecting the optic nerve.
\end{abstract}

Key words: Non-arteritic Anterior Ischemic Optic Neuropathy (NA-AION); Mean Platelet Volume (MPV), Red Cell Distribution Width (RDW), Neutrophil/Lymphocyte Ratio (NLR); derived NLR (dNLR)

\section{Introduction}

Non-arteritic anterior ischemic optic neuropathy (NA-AION) is the most frequent form of acute optic neuropathy in individuals aged over 50 years, affecting between 2.3 and 10.2 people per 100,000 population annually in the U.S. and accounting for up to 6,000 new cases every year $[1,2]$.
NA-AION is a multifactorial disease. Systemic hypertension, diabetes mellitus, atherosclerosis, and a crowded optic disc are well-known risk factors [3, 4]. Former reports have suggested that systemic and local inflammation may affect the pathogenesis of NA-AION, by inducing both atherosclerosis and 
hypercoagulability [5-11]. Inflammation is the result of complex interactions between the immune and inflammatory system, which includes neutrophils, macrophages, and lymphocytes. The absolute counts of white blood cells (WBC), neutrophils, and lymphocytes, and their ratios can be reliable indicators of chronic inflammation [12]. Neutrophil/lymphocyte ratio (NLR) has become an important biomarker of systemic inflammation and an independent prognostic factor in some chronic diseases and solid malignancies [12]. Furthermore, recent studies have reported higher NLR values in NA-AION patients $[10,13,14]$.

The role of platelets is crucial in thrombo-occlusive disorders, such as ischemic and hemorrhagic stroke [15] and retinal vein occlusion (RVO) [16, 17]. Recent studies have suggested that platelets may also been implicated in the pathogenesis of NA-AION [14, 18]. Mean platelet volume (MPV) is an important marker of the production rate and size of platelets and has been correlated to platelet activation [19]. Larger platelets are metabolically more reactive and aggregate more easily.

The purpose of this study was to evaluate the role of complete blood cell count (CBC) dimensional measures, such as MPV and red cell distribution width (RDW), and CBC-combined indices, including NLR, derived NLR [dNLR = neutrophils/(white blood cells - neutrophils)], and platelet/lymphocyte ratio (PLR), in NA-AION.

\section{Methods}

This was a retrospective case-control study, including 37 newly diagnosed NA-AION patients and 37 sex- and age-matched subjects with no evidence of NA-AION, examined at the Ophthalmology Unit, Department of Medical, Surgical and Experimental Sciences, University of Sassari, Sassari, Sardinia, Insular Italy, in 2017-2018. To ensure appropriate matching, patients of non-Sardinian descent were excluded.

Ethical approval was waived by the local Ethics Committee of the Azienda Ospedaliero-Universitaria di Sassari, Sassari, Italy, in view of the retrospective nature of the survey, which was conducted in compliance with the tenets of the Declaration of Helsinki. Each participant received detailed information and provided informed consent before inclusion.

The inclusion criterion for the case group was the diagnosis of NA-AION. This diagnosis was established by the history of sudden visual loss together with optic disc swelling, characteristic visual field defects, and typical fluorescein angiography features. The time span for considering the patients as "newly" diagnosed was ten days. All NA-AION patients underwent a complete eye examination, consisting of best corrected visual acuity (BCVA), slit-lamp evaluation, applanation tomometry, fundoscopy, fluorescein angiography, and automated visual field examination (Humphrey Field Analyzer 30-2 test). Visual field defects were classified by a team of readers (A.P. and P.C.). Medical conditions, including diabetes mellitus, systemic hypertension, increased blood cholesterol levels, cardio-vascular status (presence of angina and/or myocardial infarction), and cerebro-vascular status (presence of transient ischemic attacks and/or stroke) were recorded. Exclusion criteria included non-Sardinian descent and evidence of any other neurological, systemic, or ophthalmic disorder that could account for optic disc swelling and visual loss.

One sex- and aged-matched control per each case was randomly selected from the cataract registry. Subjects with present or past history of AION, RVO, or retinal artery occlusion were excluded. All controls underwent a complete eye examination, consisting of BCVA, slit-lamp biomicroscopy, applanation tonometry, and dilated fundus evaluation. The same medical conditions as for NA-AION patients were also recorded.

Definitions of systemic hypertension, diabetes, and hypercholesterolemia have been reported previously [20].

On the same day of NA-AION diagnosis, blood samples were collected by standard venipuncture, and blood cell counts were made using an automatic blood counter Cell-Dyn Sapphire (Abbott Diagnostics, Santa Clara, CA, USA) in a certified laboratory. Erythrocyte sedimentation rate (ESR) was also measured using standard techniques. Blood sample collection for routine tests is standard procedure for all AION and cataract patients.

Blood cell counts, dimensional indices (MPV, RDW), and combined indices, such as NLR, dNLR, and PLR, were evaluated.

Results were reported as mean \pm standard deviation (SD) or median and interquartile range (IQR) values, as appropriate. Shapiro-Wilk test was used to assess variable distribution. Student's t-test, Welch-test for data with unequal variances, or Mann-Whitney rank sum test was used to assess statistical differences between groups.

The ability of platelet number, MPV, RDW, NLR, and dNLR to predict NA-AION was determined using receiver operating characteristics (ROC) curve analysis. The optimal cut-off for sensitivity and specificity was calculated by maximizing the Youden's index.

Multivariate logistic regression models, 
including all covariates found to be significantly associated with NA-ION in univariate logistic regression analysis (i.e., platelet number, $\mathrm{MPV}, \mathrm{RDW}$, NLR, and dNLR) were used. NLR and dNLR were assessed separately to avoid collinearity. Odds ratios (ORs) and 95\% confidence intervals (CIs) were determined.

$P$ values $\leq 0.05$ were considered to be statistically significant. Statistical analysis was performed using MedCalc for Windows, version 15.4 64 bit (MedCalc Software, Ostend, Belgium).

The attributable risk of the association between NA-AION and RDW was calculated. For this purpose, the upper limit of the reference range for RDW $(14.5 \%)$ was used.

\section{Results}

One percent of the patients with NA-AION and $1 \%$ of the controls eligible for this retrospective survey declined to participate. The main reason was "not interested".

The study group included 37 newly diagnosed NA-AION patients (23 men, 14 women; mean age: $65.6 \pm 12.4$ years). No patient reported pain with eye movements before visual loss. All eyes with NA-AION had no vision improvement over time. Automated perimetry revealed an altitudinal defect in $22(60 \%)$ eyes and generalized depression in $15(40 \%)$. All affected eyes had extensive leakage of fluorescein in the swollen portion of the optic disc.

Demographics, medical history details, and CBC results of NA-AION patients and cataract controls with no evidence of NA-AION are presented in Table 1. NA-AION patients and controls had similar percentages of systemic hypertension and hypercholesterolemia. Similarly, there were no significant differences in WBC, lymphocyte, and neutrophil counts and ESR values. Conversely, NA-AION patients showed significantly lower mean values of platelet number, significantly higher rates of diabetes mellitus and cerebro/cardiovascular disease, and significantly higher median values of MPV, RDW, NLR and dNLR. These values were acutely altered around the time of NA-AION onset.

Table 2 shows ROC curve analysis results for platelet count, MPV, RDW, NLR, and dNLR. The area under the curve (AUC) values disclosed a good specificity only for dNLR, whereas all the indices analyzed showed a relatively poor sensitivity.

Results of multivariate logistic regression models, including platelet number, MPV, RDW, and NLR or dNLR as covariates are presented in Table 3. A statistically significant relationship between RDW and NA-AION ( $p \leq 0.05)$ was found.
RDW accounted for $33 \%$ of the attributable risk for NA-AION.

\section{Discussion}

NA-AION is a leading cause of acute loss of vision in individuals aged 55 to 70 years. This condition is thought to be the result of circulatory failure within the optic disc. Many systemic disorders, which may reduce optic disc perfusion, have been reported as risk factors. Among these there are systemic hypertension [3, 4, 21], diabetes mellitus [3, 4, 22], hypercholesterolemia [4, 23], ischemic heart disease [3], acute blood loss [24], and marked nocturnal arterial hypotension, especially in patients taking anti-hypertensive medications [25]. Furthermore, a crowded optic disc may be a predisposing factor for NA-AION, either by mechanically impairing the axoplasmic flow or amplifying the microvascular compression secondary to optic disc swelling of any cause [26]. On the other hand, Glucose-6-Phosphate Dehydrogenase (G6PD) deficiency may have a protective effect against the development of NA-AION [27].

Table 1. Demographic data, medical history details, and blood cell count results of patients with non-arteritic anterior ischemic optic neuropathy (NA-AION) and cataract controls without NA-AION

\begin{tabular}{|c|c|c|c|}
\hline & $\begin{array}{l}\text { NA-AION } \\
\text { patients (37) }\end{array}$ & Controls (37) & p-value \\
\hline $\begin{array}{l}\text { Age, years, mean } \pm \text { Standard } \\
\text { Deviation }(S D)\end{array}$ & $65.6 \pm 12.4$ & $65.6 \pm 12.4$ & 0.99 \\
\hline Gender (males/females) & $23 / 14$ & $23 / 14$ & 1 \\
\hline Diabetes, n (\%) & $5(13.5)$ & $1(2.7)$ & 0.09 \\
\hline Hypertension, n (\%) & $17(46.3)$ & $17(46.3)$ & 1.00 \\
\hline Hypercholesterolemia, n (\%) & $10(27.0)$ & $12(32.4)$ & 0.61 \\
\hline $\begin{array}{l}\text { Cerebro-/Cardio-vascular } \\
\text { disease, } \mathrm{n}(\%)\end{array}$ & $9(24.3)$ & $3(8.11)$ & 0.06 \\
\hline $\begin{array}{l}\text { White Blood cell Count, } \times \\
10^{9} / \mathrm{L} \text {, median (IQR) }\end{array}$ & $6.60(5.93-8.33)$ & $6.67(6.03-8.44)$ & 0.82 \\
\hline $\begin{array}{l}\text { Lymphocytes, } \times 10^{9} / \mathrm{L}, \\
\text { mean } \pm \mathrm{SD}\end{array}$ & $1.88 \pm 0.55$ & $2.14 \pm 0.67$ & 0.24 \\
\hline $\begin{array}{l}\text { Neutrophils, } \times 10^{9} / \mathrm{L}, \\
\text { median (IQR) }\end{array}$ & $4.00(3.28-5.15)$ & $3.80(3.35-5.05)$ & 0.53 \\
\hline $\begin{array}{l}\text { Platelet number, } \times 10^{9} / \mathrm{L}, \\
\text { mean } \pm \text { SD }\end{array}$ & $221 \pm 48$ & $248 \pm 56$ & 0.03 \\
\hline $\begin{array}{l}\text { Mean Platelet Volume [MPV] } \\
\text { (fl), median (IQR) }\end{array}$ & $8.20(7.60-8.90)$ & $7.60(7.05-8.25)$ & 0.013 \\
\hline $\begin{array}{l}\text { Red cell Distribution Width } \\
\text { [RDW] }(\%), \text { median (IQR) }\end{array}$ & $13.0(12.0-14.5)$ & $12.0(11.6-13.0)$ & 0.015 \\
\hline $\begin{array}{l}\text { Neutrophil/Lymphocyte Ratio } \\
\text { (NLR), median (IQR) }\end{array}$ & $2.50(1.77-3.06)$ & $1.95(1.43-2.49)$ & 0.03 \\
\hline $\begin{array}{l}\text { Derived NLR (dNLR), } \\
\text { median (IQR) }\end{array}$ & $1.73(1.31-2.07)$ & $1.36(1.07-1.69)$ & 0.01 \\
\hline $\begin{array}{l}\text { Platelet/Lymphocyte Ratio } \\
\text { (PLR), median (IQR) }\end{array}$ & 116 (98-147) & $117(97-152)$ & 0.97 \\
\hline $\begin{array}{l}\text { Erythrocyte sedimentation rate } \\
(\mathrm{mm} / \mathrm{hr}), \text { mean } \pm \mathrm{SD}\end{array}$ & $22.5 \pm 16.4$ & $19.9 \pm 14.8$ & 0.48 \\
\hline
\end{tabular}

The exact pathophysiological mechanisms underlying NA-AION are still unclear. The rapid onset, stable course with no or minimal visual 
improvement, and association with circulatory risk factors are suggestive of a vascular origin. According to the most reliable theory, impairment of the optic disc circulation, aggravated by axonal crowding, eventually reaches a point at which insufficient oxygen levels cause ischemia and disc edema [28]. A vicious circle of ischemia, swelling of axons, microvascular compression, and further ischemia leads to progressive optic nerve head damage. Nocturnal arterial hypotension contributes to the worsening of ischemia [25].

Table 2. Receiver operating characteristics (ROC) curves and prognostic accuracy of platelet number, mean platelet volume (MPV), red cell distribution width (RDW), neutrophil/lymphocyte ratio (NLR), and derived NLR (dNLR) in non-arteritic anterior ischemic optic neuropathy

\begin{tabular}{|c|c|c|c|c|c|c|}
\hline Marker & AUC & $95 \% \mathrm{CI}$ & $\mathrm{p}$ value & Cut-off & Sensitivity & Specificity \\
\hline $\begin{array}{l}\text { Platelet } \\
\text { number }\end{array}$ & 0.657 & $0.537-0.763$ & 0.016 & $<240$ & 70.27 & 62.16 \\
\hline MPV & 0.669 & $0.549-0.775$ & 0.008 & $>7.8$ & 62.16 & 63.89 \\
\hline RDW & 0.664 & $0.544-0.769$ & 0.009 & $>12$ & 70.27 & 59.46 \\
\hline NLR & 0.645 & $0.526-0.753$ & 0.026 & $>2.11$ & 62.16 & 67.57 \\
\hline dNLR & 0.671 & $0.552-0.776$ & 0.008 & $>1.72$ & 54.05 & 81.08 \\
\hline
\end{tabular}

Table 3. Multivariate logistic regression analysis models, showing odds ratios for non-arteritic anterior ischemic optic neuropathy (NA-AION = 37)

\begin{tabular}{|c|c|c|c|c|c|c|}
\hline & \multicolumn{3}{|c|}{ Model with NLR } & \multicolumn{3}{|c|}{ Model with dNLR } \\
\hline & OR & $\begin{array}{l}95 \% \text { Confidence } \\
\text { Interval }\end{array}$ & $\begin{array}{l}\mathrm{p} \\
\text { value }\end{array}$ & OR & $\begin{array}{l}\text { 95\% Confidence } \\
\text { Interval }\end{array}$ & $\begin{array}{l}\mathrm{p} \\
\text { value }\end{array}$ \\
\hline RDW & 1.453 & $0.991-2.131$ & 0.05 & 1.474 & $1.0001-2.173$ & 0.049 \\
\hline Platelets & 0.996 & $0.985-1.007$ & 0.44 & 0.996 & 0.985-1.007 & 0.48 \\
\hline MPV & 1.354 & $0.814-2.252$ & 0.24 & 1.375 & $0.821-2.302$ & 0.23 \\
\hline NLR & 1.604 & $0.873-2.948$ & 0.13 & -- & -- & -- \\
\hline dNLR & -- & -- & -- & 2.63 & $0.958-7.220$ & 0.061 \\
\hline
\end{tabular}

Number of controls: 37. RDW: red cell distribution width; MPV: mean platelet

volume; NLR: neutrophil/lymphocyte ratio; dNLR: derived NLR.

Platelets play a critical role in thrombo-occlusive disease. MPV is an important marker of platelet size and activity. Larger platelets produce more thromboxane A2, express more glycoprotein $\mathrm{Ib}$ and glycoprotein $\mathrm{IIb} / \mathrm{III} a$ receptors, and aggregate more easily $[19,29]$.

In our survey, patients with NA-AION showed a significantly lower platelet count and significantly larger platelets than controls. Former investigations have reported a correlation between NA-AION and increased MPV [11, 13, 14, 18], a result consistent with our study. Furthermore, Stiebel-Kalish et al. [30], in a systematic review and meta-analysis on aspirin use after NA-AION, concluded that there is weak evidence showing a beneficial effect of acetylsalicylic acid in preventing NA-AION in the second eye. On the whole, these findings support the idea that platelets may be somehow involved in the pathogenesis of NA-AION. However, while platelets do play an important role in thrombo-occlusive disease, their role in NA-AION is less clear. According to Hayreh [31], in the typical NA-AION, which is a hypotensive disorder, there is no scientific rationale for aspirin use in preventing recurrences of NA-AION, if not for a small number of cases due to true embolism, where it may be beneficial [31].

Changes in CBC dimensional indices, such as MPV and RDW, reflect inflammation severity in several disorders, because systemic inflammation not only affects the survival of platelets and red blood cells, but also deforms their membranes [32]. RDW is an index measuring variation in red blood cell size or volume (anisocytosis). Generally, it is expressed as the ratio between the standard deviation of erythrocyte volume and mean corpuscular volume (MCV), multiplied by 100 . RDW has historically been used to help classify anemia. However, it has recently been implicated in subclinical inflammation, as well as in other human diseases different from anemia, including chronic heart failure, myocardial infarction, stroke, liver impairment, pregnancy complications, and NA-AION [14, 33, 34]. In our investigation, we observed that RDW values were higher in NA-AION patients than in controls.

NLR is a broadly accessible, easy to calculate, and cheap inflammatory biomarker. Clinical evidence suggests that it may predict disease development, progression, and prognosis and response to treatment. A relationship between NLR and disease activity and outcome has been observed in several malignancies and chronic inflammatory disorders, including arthritis, diabetes mellitus, systemic hypertension, and chronic obstructive pulmonary disease [12, 35-38]. An association between the severity of diabetic retinopathy and NLR has been documented [39], whereas contrasting data have been reported about the role of NLR in AMD [40-42].

In this study, patients with newly diagnosed NA-AION had significantly higher NLR and dNLR values than cataract controls with no evidence of present or past NA-AION. Likewise, in a retrospective study on 45 NA-AION patients and 50 age- and sex-matched controls, Polat et al. [13] found that NA-AION patients had significantly higher values of NLR. A similar result was reported by Gunes et al. [10] in a survey comparing 56 NA-AION patients with 56 healthy subjects. These authors also found that ROC curves for NLR predicted NA-AION development with a sensitivity of $85 \%$ and specificity of $41 \%$ [10]. Conversely, Inanc et al. [11] observed no correlation between NLR and NA-AION.

In our study, dNLR showed a higher statistical significance than NLR $(p=0.01$ vs $p=0.03)$. 
Furthermore, AUC values disclosed a higher specificity for dNLR ( $81 \%$ vs $68 \%)$. Overall, these findings seem to support the possibility that dNLR might be a more reliable biomarker than NLR in patients with NA-AION.

Multivariate logistic regression models disclosed a significant correlation only between increased RDW and NA-AION $(p \leq 0.05)$. The attributable risk of the association between NA-AION and RDW was 33\%. The clinical meaning of these findings is not clear. Recently, Ozkok et al. [43] have reported that RDW was significantly higher and correlated significantly with baseline and final visual acuity in RVO patients. These authors postulated that RDW may represent an integrative measure of a multifactorial disease process, involving oxidative stress, chronic inflammation, and endothelial dysfunction, which contribute to RVO pathogenesis. Possibly, this may also be the case in NA-AION.

Our study has some intrinsic limitations, such as the retrospective nature and case-control design. This type of design usually do not allow to determine the temporal relationship between exposure and disease; therefore, it cannot be clearly determined whether the higher values of MPV, RDW, NLR, and dNLR are possible causes of NA-AION or simply correlates of other factors associated with NA-AION. Prospective studies may provide a more reliable evidence to look for a potential cause-effect relationship between these dimensional and CBC-combined indices and NA-AION. Another limitation of this study is the fact that the cases and controls were not matched for certain parameters which might have affected $C B C$ indices, such as body mass index, diabetic control, anemia, anti-hypertensive medications, and smoking history. Furthermore, we do not know how many patients had a crowded optic disc, a known risk factor for NA-AION, because data about optic disc size were incomplete. Last, but not least, our survey was performed on a relatively small, genetically homogeneous group of subjects of Sardinian ancestry. Consequently, our results might not be extended to NA-AION patients of non-Sardinian descent.

To sum up, our results suggest that RDW may be a potential disease biomarker in patients with NA-AION. However, high-quality cohort studies are warranted before any definitive conclusion on the putative role of increased RDW on NA-AION can be drawn.

\section{Acknowledgements}

This manuscript was in part presented as a Scientific Poster (\#5244) at the 22nd European Association for Vision and Eye Research (EVER) Meeting, Nice (France), October 17-19, 2019.
Pinna A, Porcu T, Marzano J, Boscia F, Paliogiannis P, Carru C, Zinellu A (2019), Complete blood cell count indexes in non-arteritic anterior ischemic optic neuropathy. Abstracts from the European Association for Vision and Eye Research Conference, published in Acta Ophthalmologica, Volume 97, Issue S263. Chichester (UK), Wiley Online Library, John Wiley \& Sons, Inc.

\section{Author contributions}

All authors contributed to the study conception and design. Material preparation, data collection and analysis were performed by Antonio Pinna, Paola Carlino, Rita Serra, Stefano Dore, and Angelo Zinellu. The first draft of the manuscript was written by Antonio Pinna and all authors commented on previous versions of the manuscript. All authors read and approved the final manuscript.

\section{Competing Interests}

Funding: Antonio Pinna has received research grants by:

1) "Regione Autonoma della Sardegna," Italy, (\#RAS 2010, CRP-25871 ANTONIO PINNA);

2) University of Sassari, Italy. Fondo di Ateneo per la ricerca, annualità 2020. Rep. 2465/2020, Prot. 0097985 del 01/09/2020.

The funding organizations had no role in the design or conduct of this research.

The remaining authors (Paola Carlino, Rita Serra, Francesco Boscia, Stefano Dore, Ciriaco Carru, and Angelo Zinellu) declare that they have no conflict of interest.

\section{References}

1. Johnson LN, Arnold AC. Incidence of nonarteritic and arteritic anterior ischemic optic neuropathy. J Neuro-Ophthalmol 1994; 14: 38-44.

2. Hattenhauer MG, Leavitt JA, Hodge DO, et al. Incidence of nonarteritic ischemic optic neuropathy. Am J Ophthalmol 1997; 123: 103-107.

3. Hayreh SS, Joos KM, Podhajsky PA, et al. Systemic diseases associated with nonarteritic anterior ischemic optic neuropathy. Am J Ophthalmol 1994; 118: 766-780.

4. Deramo VA, Sergott RC, Augsburger JJ, et al. Ischemic optic neuropathy as the first manifestation of elevated cholesterol levels in young patients. Ophthalmology 2003; 110: 1041-1045.

5. Goldenberg-Cohen N, Kramer M, Bahar I, et al. Elevated plasma levels of interleukin 8 in patients with acute anterior ischaemic optic neuropathy. Br J Ophthalmol 2004; 88: 1538-1540.

6. Hayreh SS. Ischemic optic neuropathy. Prog Retin Eye Res 2009; 28: 34-62.

7. Zhang C, Guo Y, Miller NR, et al. Optic nerve infarction and post-ischemic inflammation in the rodent model of anterior ischemic optic neuropathy (rAION). Brain Res 2009; 1264: 67-75.

8. Salgado C, Vilson F, Miller NR, et al. Cellular inflammation in nonarteritic anterior ischemic optic neuropathy and its primate model. Arch Ophthalmol 2011; 129: 1583-1591.

9. Slater BJ, Vilson FL, Guo Y, et al. Optic nerve inflammation and demyelination in a rodent model of nonarteritic anterior ischemic optic neuropathy. Invest Ophthalmol Vis Sci 2013; 54: 7952-7961.

10. Gunes A, Yigit M, Tok L, et al. Neutrophil to lymphocyte ratio in patients with nonarteritic anterior ischemic optic neuropathy. Korean J Ophthalmol 2017; 31: 159-164.

11. Inanc $\mathrm{M}$, Tekin $\mathrm{K}$, Budakoglu $\mathrm{O}$, et al. Could platelet indices and neutrophil to lymphocyte ratio be new biomarkers for differentiation of arteritic anterior ischemic neuropathy from non-arteritic type? Neuro-ophthalmol 2018; 42: 287-294. 
12. Paliogiannis $\mathrm{P}$, Fois AG, Sotgia $\mathrm{S}$, et al. Neutrophil to lymphocyte ratio and clinical outcomes in COPD: recent evidence and future perspectives. Eur Respir Rev 2018; 27(147): 170113.

13. Polat $\mathrm{O}$, Yavaş GF, İnan S, et al. Neutrophil-to-Lymphocyte Ratio as a marker in patients with Non-arteritic Anterior Ischemic Optic Neuropathy. Balkan Med J 2015; 32: 382-387.

14. Pinna A, Porcu T, Marzano J, et al. Complete blood cell count indexes in nonarteritic anterior ischemic optic neuropathy. Abstract from the 2019 European Association for Vision and Eye Research Conference. Acta Ophthalmol 2019; 97: S26.

15. Du J, Wang Q, He B, et al. Association of mean platelet volume and platelet count with the development and prognosis of ischemic and hemorrhagic stroke. Int J Lab Hematol 2016; 38: 233-239.

16. Onder HI, Kilic AC, Kaya M, et al. Relation between platelet indices and branch retinal vein occlusion in hypertensive patients. Indian J Ophthalmol 2013 61: 160-162.

17. Sahin A, Sahin M, Yüksel H, et al. The mean platelet volume in patients with retinal vein occlusion. J Ophthalmol 2013; 236371.

18. Sahin M, Sahin A, Elbey B, et al. Mean platelet volume in patients with nonarteritic anterior ischemic optic neuropathy. J Ophthalmol 2016: 1051572.

19. Park Y, Schoene N, Harris W. Mean platelet volume as an indicator of platelet activation: methodological issues. Platelets 2002; 13: 301-306.

20. Pinna A, Contini EM, Carru C, et al. Glucose-6-phosphate dehydrogenase deficiency and diabetes mellitus with severe retinal complications in a Sardinian population, Italy. Int J Med Sci 2013; 10: 1907-1913.

21. Hayreh SS, Jonas JB, Zimmerman MB. Nonarteritic anterior ischemic optic neuropathy and tobacco smoking. Ophthalmology 2007; 114: 804-809.

22. Jacobson DM, Vierkant RA, Belongia EA. Nonarteritic ischemic optic neuropathy. A case-control study of potential risk factors. Arch Ophthalmol 1997; 115: 1403-1407.

23. Talks SJ, Chong NH, Gibson JM, et al. Fibrinogen, cholesterol and smoking as risk factors for nonarteritic anterior ischemic optic neuropathy. Eye 1995; 9: $85-88$

24. Hayreh SS. Anterior ischemic optic neuropathy. VIII. Clinical features and pathogenesis of post-hemorrhagic amaurosis. Ophthalmology 1987; 94: 1488-1502.

25. Hayreh SS, Zimmerman MB, Podhajsky P, et al. Nocturnal arterial hypotension and its role in optic nerve head and ocular ischemic disorders. Am J Ophthalmol 1994; 117: 603-624.

26. Beck RW, Servais GE, Hayreh SS. Anterior ischemic optic neuropathy. IX. Cup-to-disc ratio and its role in pathogenesis. Ophthalmology 1987; 94: 1503-1508.

27. Pinna A, Solinas G, Masia C, et al. Glucose-6-phosphate dehydrogenase (G6PD) deficiency in nonarteritic anterior ischemic optic neuropathy in a Sardinian population, Italy. Invest Ophthalmol Vis Sci 2008; 49: 1328-1332.

28. Arnold AC. Pathogenesis on nonarteritic anterior ischemic optic neuropathy. J Neuro-Ophthalmol 2003; 23: 157-163.

29. Giles H, Smith REA, Martin JF. Platelet glycoprotein IIb-IIIa and size are increased in acute myocardial infarction. Eur J Clin Invest 1994; 24: 69-72.

30. Stiebel-Kalish H, Hasanreisoglu M, Leibovici L. Aspirin following nonarteritic ischaemic optic neuropathy - a systematic review and metanalysis. Neuro-ophthalmol 2010; 34: 14-19.

31. Hayreh SS. The role of aspirin in non-arteritic ischaemic optic neuropathy. Neuro-ophthalmol 2010; 34: 1-5.

32. Hu ZD, Sun Y, Guo J, et al. Red blood cell distribution width and neutrophil/ lymphocyte ratio are positively correlated with disease activity in primary Sjögren's syndrome. Clin Biochem 2014; 47: 287-290.

33. Lippi G, Plebani M. Red blood cell distribution width (RDW) and human pathology. One size fits all. Clin Chem Lab Med 2014; 52: 1247-1249.

34. Paliogiannis P, Zinellu A, Mangoni AA, et al. Red blood cell distribution width in pregnancy: a systematic review. Biochem Med (Zagreb) 2018; 28(3): 030502 .

35. Dolan RD, Lim J, McSorley ST, et al. The role of the systemic inflammatory response in predicting outcomes in patients with operable cancer: Systematic review and meta-analysis. Sci Rep 2017; 7(1): 16717.

36. Dolan RD, McSorley ST, Horgan PG, et al. The role of the systemic inflammatory response in predicting outcomes in patients with advanced inoperable cancer: Systematic review and meta-analysis. Crit Rev Oncol Hematol 2017; 116: 134-146.

37. Fu H, Qin B, Hu Z, et al. Neutrophil- and platelet-to-lymphocyte ratios are correlated with disease activity in rheumatoid arthritis. Clin Lab 2015; 61: 269-273.

38. Mertoglu C, Gunay M. Neutrophil-Lymphocyte ratio and PlateletLymphocyte ratio as useful predictive markers of prediabetes and diabetes mellitus. Diabetes Metab Syndr 2017; 11(Suppl 1): S127-S131.

39. Ulu SM, Dogan M, Ahsen A, et al. Neutrophil-to-lymphocyte ratio as a quick and reliable predictive marker to diagnose the severity of diabetic retinopathy. Diabetes Technol Ther 2013; 15: 942-947.

40. Ilhan N, Daglioglu MC, Ilhan O, et al. Assessment of Neutrophil/Lymphocyte Ratio in patients with age-related macular degeneration. Ocul Immunol Inflamm 2015; 23: 287-290.

41. Sengul EA, Artunay O, Kockar A, et al. Correlation of neutrophil/lymphocyte and platelet/lymphocyte ratio with visual acuity and macular thickness in age-related macular degeneration. Int J Ophthalmol 2017; 10: 754-759.
42. Pinna A, Porcu T, D'Amico-Ricci G, et al. Complete blood cell count-derived inflammation biomarkers in men with age-related macular degeneration. Ocul Immunol Inflamm 2019; 27: 932-936.

43. Ozkok A, Nesmith BLW, Schaal S. Association of red cell distribution width values with vision potential in retinal vein occlusion. Ophthalmol Retina 2018; 2: $582-586$ 